\title{
Fecundability among women with type 1 and type 2 diabetes in the Norwegian Mother and Child Cohort Study
}

\author{
K. W. Whitworth • D. D. Baird • L. C. Stene • \\ R. Skjaerven • M. P. Longnecker
}

Received: 21 July 2010 /Accepted: 12 November 2010 /Published online: 19 December 2010

(C) Springer-Verlag (outside the USA) 2010

\begin{abstract}
Aims/Hypothesis We assessed the effects of type 1 diabetes and type 2 diabetes on fecundability (as manifest by increased time-to-pregnancy [TTP]) in a large cohort of pregnant women.

Methods This study is based on the Norwegian Mother and Child Cohort Study. Members of this large cohort were enrolled early in pregnancy and asked about TTP and other factors. Among the 58,004 women included in the analysis, we identified 221 cases of type 1 diabetes and 88 cases of type 2 diabetes using the Medical Birth Registry of Norway. A logistic analogue of the proportional probability
\end{abstract}

Electronic supplementary material The online version of this article (doi:10.1007/s00125-010-2003-6) contains supplementary material, which is available to authorised users.

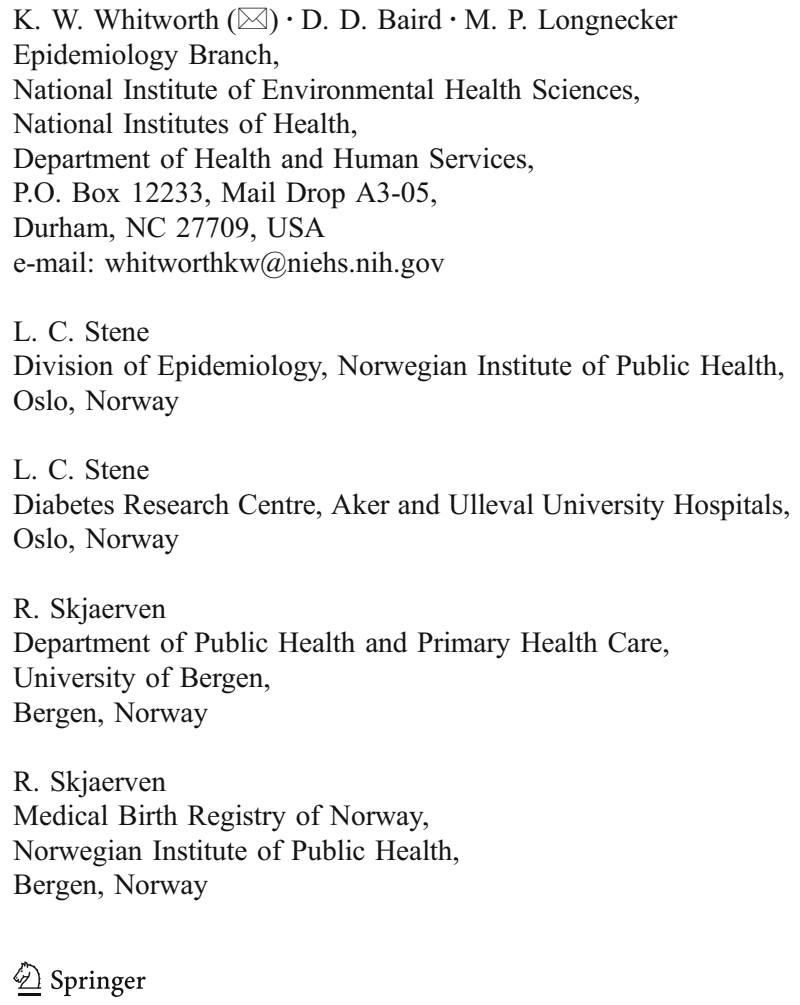

model, a Cox-like discrete-time model, was used to compute fecundability odds ratios (FORs) and $95 \%$ CI for type 1 diabetes and type 2 diabetes, adjusted for maternal age and prepregnancy BMI.

Results Compared with non-diabetic women, the adjusted FOR for women with type 1 diabetes was 0.76 (95\% CI 0.64-0.89) and the adjusted FOR for women with type 2 diabetes was 0.64 (95\% CI 0.48-0.84). These FORs did not change substantively and remained statistically significant after excluding women with irregular menstrual cycles and accounting for cycle length.

Conclusions/Interpretation The results from the present study provide evidence of substantially decreased fecundability for women with type 1 and type 2 diabetes, even among those with a normal menstrual cycle.

Keywords Fecundability · Pregestational diabetes . Pregnancy · Time-to-pregnancy - Type 1 diabetes . Type 2 diabetes
Abbreviations
FOR Fecundability odds ratio
GnRH Gonadotropin-releasing hormone
HPO Hypothalamic-pituitary-ovarian
MBRN Medical Birth Registry of Norway
$\mathrm{MoBa}$ Norwegian Mother and Child Cohort Study
NorPD Norwegian Prescription Database
TTP Time-to-pregnancy

\section{Introduction}

Women with type 1 diabetes may experience delayed onset of menarche, early menopause and menstrual cycle irregularities [1]. For example, a study of Danish women reported a higher prevalence of menstrual irregularities 
among women with type 1 diabetes (21.6\%) compared with controls $(10.8 \%)$ [2]. Further, a study conducted in the USA found that women with type 1 diabetes had up to twice the frequency of menstrual irregularities compared with controls [3]. Menstrual cycle irregularities among women with type 2 diabetes have been less well studied. However, the Nurses' Health Study reported an increased risk of developing type 2 diabetes among women with long cycles (RR 2.0 [95\% CI 1.6-2.7]) or very irregular cycles (RR 1.5 [95\% CI 1.3-1.8]) [4].

Studies have also reported increased rates of spontaneous abortion among women with type 1 diabetes [5], and of perinatal mortality and birth defects among women with either type 1 or type 2 diabetes $[6,7]$.

Due to the reproductive abnormalities experienced by women with diabetes, a reduction in fecundability (the ability to conceive) is expected. Yet data that directly address the effects of diabetes on women's fecundability or fertility are scarce [1]. In Sweden, the live birth rate among women first hospitalised with type 1 diabetes since 1985 has been normal [8]. Live birth rate, however, reflects fertility rather than fecundability. Because fecundability is the odds of conception (regardless of pregnancy outcome), live birth rate is a relatively insensitive measure of fecundability. For example, conceptions leading to spontaneous abortions are counted towards fecundability but are not reflected in the live birth rate. Additionally, women's family-planning decisions can alter a population's fertility rate (by lowering the number of live births), even if the population is fecund. Therefore, data regarding pregnancy attempts are necessary to make conclusions regarding fecundability. In the present study, we examined the effects of type 1 diabetes and type 2 diabetes on fecundability in a large study of pregnant women.

\section{Methods}

This study was based on the Norwegian Mother and Child Cohort Study (MoBa), conducted by the Norwegian Institute of Public Health [9]. A total of 235,577 women in Norway were invited to participate in MoBa. Women were enrolled from 1999-2008 and received an invitation to participate in MoBa through the mail with their notice of an appointment for a routine ultrasound, given to all women in Norway at about week $17-18$ of gestation. The participation rate was $38.5 \%$, resulting in 90,697 women who participated in MoBa and 106,977 pregnancies. Additional details can be found at www.fhi.no/morogbarn. MoBa was approved by the Regional Committee for Medical Research Ethics and the Norwegian Data Inspectorate. Informed consent was obtained from each participant.

Mothers completed a questionnaire at about 17 weeks of gestation. Maternal demographics (age, height, prepreg- nancy weight and education), lifestyle factors (physical activity, smoking and alcohol use 3 months before pregnancy; frequency of intercourse 1 month before pregnancy) and maternal diseases (hypo- or hyperthyroidism, fallopian tube infection, endometriosis and sexually transmitted diseases) were obtained from this questionnaire. Maternal prepregnancy BMI was constructed using the selfreported values from this questionnaire. The questionnaire also included questions related to reproductive history (cycle length, irregular cycles in the last year, parity, history of fertility treatments, and time-to-pregnancy [TTP]). Regarding TTP, women were first asked whether they planned their pregnancy. If they answered positively, they were asked a series of questions regarding how long it took them to achieve pregnancy including 'How many months did you have regular intercourse without contraception before you became pregnant?' Women chose ' $<1$ month', '1-2 months' or ' 3 months or more'. If they answered ' 3 months or more', they were further prompted to list the number of months.

Data on MoBa pregnancies were linked with data in the Medical Birth Registry of Norway (MBRN). The MBRN data were collected by the physician or midwife attending the childbirth, who had access to the pregnancy medical record [10]. Data from the MBRN were used to ascertain women's diabetes status (pregestational type 1 diabetes, pregestational type 2 diabetes, unspecified pregestational diabetes or gestational diabetes).

Eligibility for the present analysis was restricted to a woman's first pregnancy registered with $\mathrm{MoBa}$ (that is, a woman's first study pregnancy, which may not be her first lifetime pregnancy). A total of 77,820 pregnancies were eligible. Further exclusions were made as shown in Fig. 1. Thus, a total of 58,004 pregnancies, including 221 type 1 diabetic women and 88 type 2 diabetic women, were included in the analysis.

The logistic analogue of the proportional probability model, a Cox-like discrete-time model [11], was used to estimate fecundability odds ratios (FORs) and $95 \%$ CIs for associations between type 1 diabetes or type 2 diabetes and fecundability (odds of conception in one menstrual cycle). The equation for this model is given in the Electronic supplementary material [ESM]. This is a standard statistical method used in studies of fecundability [12] and was implemented using PROC LOGISTIC in SAS (SAS Institute, Cary, NC, USA). For each month that contributes to the analysis, the FOR measures the fecundability in the exposed (women with type 1 diabetes or type 2 diabetes) divided by the fecundability in the unexposed (women with no diabetes), conditional on not having conceived in any previous month. The FOR is the weighted average of the estimates across all contributing months. An FOR of $<1$ indicates reduced fecundability (increased TTP). The unit 


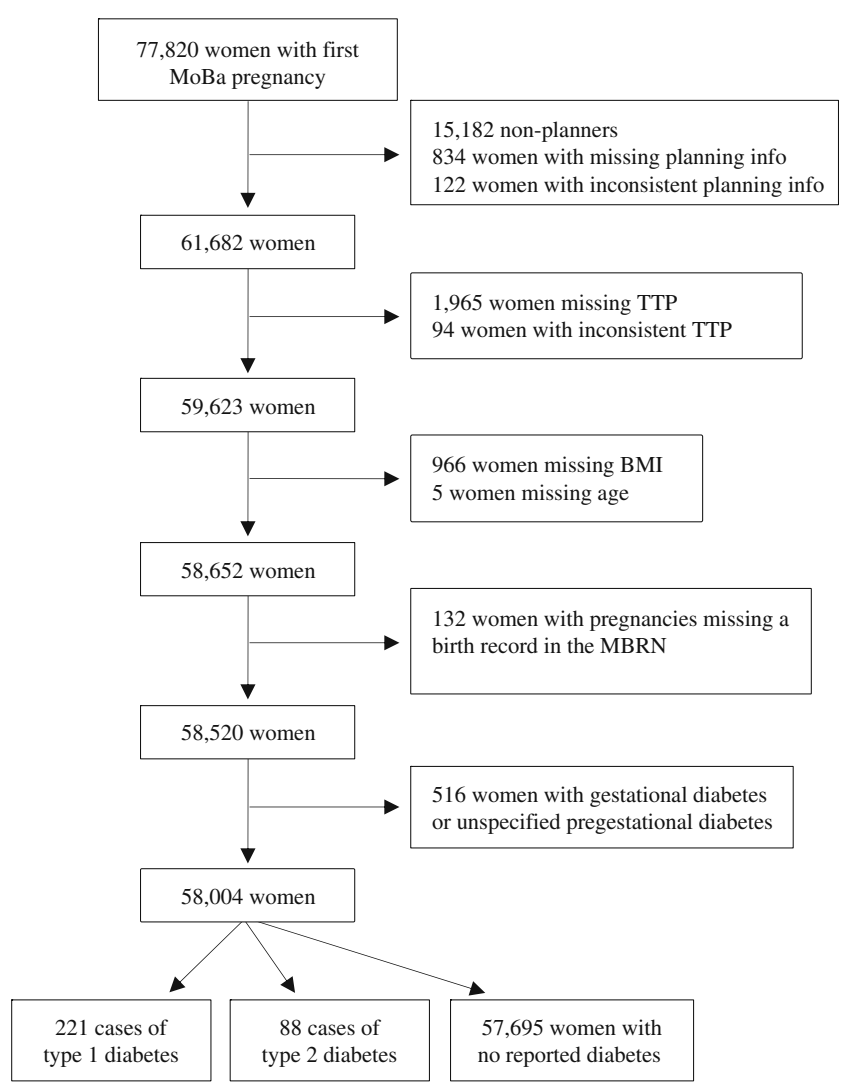

Fig. 1 Flow chart for participant inclusion based on MoBa Database Version 4.301, restricted to MoBa first pregnancies

of analysis was the woman-month and each woman contributed up to 12 months to the analysis. Women's reported TTP was used to construct an analysis dataset with multiple records per woman (one record for each month she was trying to conceive). As recommended for avoiding medical intervention bias, TTP was censored at 13 months because women are considered clinically infertile after 12 months of unsuccessful pregnancy attempts [13]. For example, if a woman reported getting pregnant after 14 months, only her first 12 (unsuccessful) months contributed to the analysis. Further, women who received infertility treatment at or before 12 months $(2.0 \%)$ had their TTP censored at TTP-1 month. Thus, if a woman reported getting pregnant after 9 months, and reported receiving fertility treatments, only her first 8 (unsuccessful) months contributed to the analysis.

Maternal age and prepregnancy BMI $\left(\mathrm{kg} / \mathrm{m}^{2}\right)$ were assumed to be important confounders and included in the model a priori. Maternal age was modelled as a continuous variable while prepregnancy BMI was modelled using its cubic form and all lower order terms (BMI, $\mathrm{BMI}^{2}$ and $\mathrm{BMI}^{3}$, all continuous). We further considered as potential confounders variables that may be associated with both diabetes and fecundability, conditional on maternal age and prepregnancy BMI. These variables include: maternal education, physical activity, smoking and alcohol use, and frequency of intercourse. A backward selection method was used whereby the additional covariates were dropped from the full model and the change in the effect estimate for diabetes and fecundability was assessed in a step-wise manner. Because the effect estimates changed by less than $10 \%$ with each deletion, none of these variables were deemed confounders. To further evaluate the potential confounding effects of maternal diseases (fallopian tube infection, endometriosis, sexually transmitted disease and hypo- or hyperthyroidism) without sacrificing degrees of freedom in the model, we examined the association between diabetes and fecundability after excluding women with these conditions (one at a time). Because the FORs for both type 1 and type 2 diabetes did not change, we concluded that these variables were not confounders. Interaction terms were fit to test effect modification between parity (yes/no) and diabetes, and age (continuous) and diabetes. To help determine whether interaction was present, we used an alpha level equal to 0.20 as a screening criterion. The $p$ values for interaction between parity and diabetes $(p=0.61$ for type 1 diabetes and $p=0.68$ for type 2 diabetes) and age and diabetes ( $p=0.44$ for type 1 diabetes and $p=0.65$ for type 2 diabetes) all fell well above this cutoff, providing no support for interaction. All analyses were completed using SAS version 9.2 (SAS Institute).

We completed two additional sub-analyses. In the first, we recalculated FORs after excluding women who reported irregular menstrual cycles; in the second, we recalculated FORs after excluding women who reported irregular menstrual cycles and we based the TTP calculation on the estimated number of cycles it took to get pregnant (instead of the number of months). For the analysis using cycle lengths, those who reported cycle length greater than 60 days $(0.2 \%)$ were excluded, which also probably excluded women with polycystic ovary syndrome.

Additional sensitivity analyses were conducted and the results are presented in ESM Fig. 1. These sensitivity analyses assessed planning bias (bias resulting from excluding women who did not plan their pregnancy and who may have higher fecundability than planners [13, 14]), medical intervention bias (bias that may result if the exposure under study is associated with the probability that women will receive successful medical help for infertility $[13,14])$ and wantedness bias (bias that occurs when women who did not plan their pregnancy respond that the pregnancy was in fact planned when asked about it in retrospect $[13,14])$.

\section{Results}

A total of 61,682 first $\mathrm{MoBa}$ pregnancies were planned compared with 15,182 unplanned pregnancies (Fig. 1). The 
distribution of diabetes among planners and non-planners was essentially the same (ESM Table 1). Planners were older, more educated, smoked less and had more infertility treatments than non-planners (ESM Table 1). While 11.2\% of women with no diabetes had a TTP of $>12$ months, $19.5 \%$ of women with type 1 diabetes and $29.6 \%$ of women with type 2 diabetes had a TTP of $>12$ months (Table 1). On average, women with type 2 diabetes were older than either women with type 1 diabetes or non-diabetic women at the time of their pregnancy. In addition, on average, women with either type 1 diabetes or type 2 diabetes had a higher prepregnancy BMI compared with non-diabetic women. Women with either type 1 or type 2 diabetes were also more likely to have irregular menstrual cycles in the 12 months before pregnancy and to receive fertility treatment for their pregnancy, compared with non-diabetic women.

The crude FOR for type 1 diabetes was 0.73 (95\% CI 0.62-0.86) and for type 2 diabetes was 0.53 (95\% CI $0.41-$ 0.70). After adjustment for age and prepregnancy BMI, the estimates were slightly attenuated: women with type 1 diabetes had an FOR of 0.76 (95\% CI 0.64-0.89) and women with type 2 diabetes had an FOR of $0.64(95 \% \mathrm{CI}$ 0.48-0.84) compared with women with no diabetes (Table 2). Based on our model, among women with average BMI $\left(24.1 \mathrm{~kg} / \mathrm{m}^{2}\right.$ in our study population) and less than 25 years of age with no diabetes, the fecundability
Table 1 Demographic factors among 58,004 women, by diabetes status

\begin{tabular}{|c|c|c|c|}
\hline Maternal characteristic & $\begin{array}{l}\text { No diabetes } \\
n=57,695\end{array}$ & $\begin{array}{c}\text { Type } 1 \text { diabetes } \\
n=221\end{array}$ & $\begin{array}{c}\text { Type } 2 \text { diabete } \\
n=88\end{array}$ \\
\hline TTP (mean, \%) & 4.4 & 5.4 & 6.5 \\
\hline$<1$ month & 23.0 & 20.8 & 17.1 \\
\hline $1-2$ months & 27.0 & 23.1 & 17.1 \\
\hline 3 months & 12.2 & 10.9 & 12.5 \\
\hline 4-12 months & 26.6 & 25.8 & 23.9 \\
\hline$>12$ months & 11.2 & 19.5 & 29.6 \\
\hline Age (mean, years) & 29.8 & 29.7 & 31.5 \\
\hline Prepregnancy BMI (mean, $\mathrm{kg} / \mathrm{m}^{2}$ ) & 24.1 & 26.3 & 28.5 \\
\hline \multicolumn{4}{|c|}{ Smoking 3 months before pregnancy (\%) } \\
\hline Daily & 17.3 & 20.0 & 21.6 \\
\hline Sometimes & 10.2 & 9.1 & 6.8 \\
\hline None & 72.3 & 71.0 & 70.5 \\
\hline Missing & 0.2 & 0.0 & 1.1 \\
\hline \multicolumn{4}{|c|}{ Highest completed years of education (\%) } \\
\hline$\leq 12$ years & 35.0 & 34.8 & 45.5 \\
\hline $13-16$ years & 40.7 & 47.5 & 40.9 \\
\hline$>16$ years & 22.4 & 16.3 & 12.5 \\
\hline Other & 2.1 & 1.4 & 1.1 \\
\hline \multicolumn{4}{|c|}{ Irregular menstrual cycles 12 months before pregnancy (\%) } \\
\hline Yes & 21.1 & 24.4 & 21.6 \\
\hline No & 78.7 & 74.7 & 78.4 \\
\hline Missing & 0.3 & 0.9 & 0.0 \\
\hline \multicolumn{4}{|c|}{ Infertility treatment for current pregnancy $(\%)$} \\
\hline Yes & 7.0 & 15.4 & 15.9 \\
\hline No & 93.0 & 84.6 & 84.1 \\
\hline \multicolumn{4}{|c|}{ Frequency of intercourse 1 month before pregnancy (\%) } \\
\hline$>2$ times per week & 48.5 & 51.1 & 45.5 \\
\hline 1-2 times per week & 35.5 & 34.9 & 31.8 \\
\hline$<1$ time per week & 14.7 & 12.2 & 19.3 \\
\hline Missing & 1.4 & 1.8 & 3.4 \\
\hline \multicolumn{4}{|l|}{ Parity $(\%)$} \\
\hline 0 & 52.4 & 59.3 & 42.1 \\
\hline 1 & 31.3 & 32.1 & 29.6 \\
\hline$>1$ & 16.4 & 8.6 & 28.4 \\
\hline
\end{tabular}


Table 2 Adjusted FORs and 95\% CIs associated with type 1 diabetes and type 2 diabetes
${ }^{a}$ All models adjusted for prepregnancy BMI and maternal age (see Methods)

\begin{tabular}{|c|c|c|c|c|c|c|}
\hline \multirow[t]{2}{*}{ Model $^{\mathrm{a}}$} & \multicolumn{3}{|c|}{ Type 1 diabetes } & \multicolumn{3}{|c|}{ Type 2 diabetes } \\
\hline & $n$ & FOR & $95 \% \mathrm{CI}$ & $n$ & FOR & $95 \% \mathrm{CI}$ \\
\hline \multicolumn{7}{|l|}{ All women } \\
\hline No diabetes & 57,695 & 1.00 & & 57,695 & 1.00 & \\
\hline Diabetes & 221 & 0.76 & $0.64-0.89$ & 88 & 0.64 & $0.48-0.84$ \\
\hline \multicolumn{7}{|c|}{ Excluding women reporting irregular menstrual cycles } \\
\hline No diabetes & 43,385 & 1.00 & & 43,385 & 1.00 & \\
\hline Diabetes & 165 & 0.80 & $0.66-0.97$ & 69 & 0.71 & $0.52-0.96$ \\
\hline \multicolumn{7}{|c|}{ Excluding women reporting irregular menstrual cycles and using cycle length to calculate TTP } \\
\hline No diabetes & 43,987 & 1.00 & & 43,987 & 1.00 & \\
\hline Diabetes & 161 & 0.81 & $0.66-0.98$ & 69 & 0.69 & $0.51-0.93$ \\
\hline
\end{tabular}

(expressed as a probability) was $26 \%$ vs a $21 \%$ fecundability for similar women with type 1 diabetes and an $18 \%$ fecundability for similar women with type 2 diabetes (data not shown).

Approximately $79 \%$ of women reported regular menstrual cycles, including $79 \%$ of women with no diabetes, $75 \%$ of women with type 1 diabetes and $78 \%$ of women with type 2 diabetes (Table 2). Once the analysis was restricted to these women, there was a slight attenuation in the FOR for women with type 1 or type 2 diabetes. Accounting for cycle length had little effect on the results. The additional sensitivity analyses addressing planning, wantedness and medical intervention biases suggested none had an important effect on the results (ESM).

\section{Discussion}

In these data, the FOR among women with type 1 diabetes was reduced by $24 \%$ and among women with type 2 diabetes it was reduced by $36 \%$ compared with nondiabetic women. Examination of fecundability among women with diabetes has not been previously reported, to our knowledge, probably because it requires a very large general population sample to include sufficient women with diabetes.

Although the mechanism through which diabetes affects fecundability is unknown, several have been proposed. Hyperglycaemic mouse models have shown that poor metabolic control during the periconception period may affect oocyte competence and decrease fecundability [15]. A recent review suggests that not only are women with type 2 diabetes less likely to obtain prepregnancy counselling than type 1 diabetic women, but diabetic women who do not seek prepregnancy counselling have decreased metabolic control [16]. Although the present study was unable to assess differences in $\mathrm{HbA}_{1 \mathrm{c}}$ levels among women with type 1 and type 2 diabetes, it is possible that poor metabolic control at the time of conception could account for the lower fecundability observed among the women with type 2 diabetes.

Type 1 and type 2 diabetes may affect fecundability through separate mechanisms. Because the preimplantation conceptus is sensitive to insulin, it may be directly affected by maternal insulin resistance. Using mouse models, Pinto et al. [17] demonstrated that excess insulin or insulin-like growth factor 1 leads to decreased levels of preimplantation GLUT 8. The decreasing levels of GLUT 8 increased apoptosis in blastocysts, increased rates of resorptions and decreased pregnancy rates [17]. Although the mouse models in these studies used glucose levels that may not be directly applicable to humans, they provide insight into how insulin resistance may affect fecundability.

Finally, alterations in the hypothalamic-pituitary-ovarian (HPO) axis could affect fecundability among both type 1 and type 2 diabetic women. Menstrual disturbances in diabetic women suggest alterations in the HPO axis [1]. Given the evidence supporting the association between diabetes and menstrual cycle irregularities, we explored the influence of cycle irregularity and cycle length on the associations described in the present study. After restricting the analysis to women with regular menstrual cycles, the FORs among women with type 1 diabetes mellitus and type 2 diabetes mellitus were attenuated but still statistically significant. We observed similar reductions in fecundability in both type 1 and type 2 diabetic women after accounting for cycle length. Past studies have documented large proportions of oligomenorrhoea among women with pregestational diabetes [2-4, 18]. Although based on small numbers, we also observed a higher proportion of longer menstrual cycles among women with diabetes compared with women without diabetes (data not shown). It appears that, regardless of menstrual disturbances (length or regularity), women with diabetes still experience a reduction in fecundability. These results, paired with observations regarding delayed menarche among type 1 diabetic 
women, may suggest a hypothalamic contribution to reduced fecundability [1].

Our study population is drawn from a cohort with a relatively large number of women with pregestational diabetes. A recent study examined the validity of diabetes diagnosis in the MBRN by cross referencing MBRN diagnoses between March 2004 and January 2007 with medications dispensed for diabetes listed in the Norwegian Prescription Database (NorPD) [19]. The authors of this study reported good sensitivity (the proportion of accurately identified cases among all true cases) for type 1 diabetes $(90 \%)$. The sensitivity of any type of diabetes (72\%) (including type 1 diabetes, type 2 diabetes and gestational diabetes) was lower, possibly as a result of the fact that the NorPD database does not capture cases of type 2 diabetes that do not require medication. Further, a separate study compared the MBRN diabetes diagnoses with medical records for 1998 and estimated that the positive predictive value of pregestational diabetes (including type 1 and type 2 diabetes) was 79.5\% (95\% CI 74.6\%-84.5\%) [20]. The mean BMI among type 1 diabetic women was higher than for those without diabetes, possibly indicating misclassification (i.e. some type 2 diabetic women may have been classified as having type 1 diabetes). However, a recent study based on Swedish medical records also reported similarly increased prepregnancy BMI among women with type 1 diabetes compared with women with no diabetes [21].

Although women were recruited during pregnancy, they were recruited early in pregnancy and are likely to have provided accurate reports of TTP and other covariates. Due to the pregnancy-based design of the MoBa study, sterile and extremely subfecund women were excluded from our sample. This may limit the generalisability of the results, but if diabetes is associated with decreased fecundability, bias resulting from including only the most fecund women in the sample will result in underestimation of the effect. Retrospective TTP studies are susceptible to planning, wantedness and medical intervention biases [13, 14]; however, when sensitivity analyses were conducted, the overall results did not change.

In the present study, we found evidence of longer TTP among women with type 1 diabetes or type 2 diabetes compared with women with no diabetes. This relationship was present even after accounting for cycle length and cycle regularity. Although menstrual and reproductive problems have been documented for women with diabetes, no study has previously demonstrated reduced fecundability directly. The reduction we observed is similar in magnitude to the FOR of obese women in a recent study [22], and we controlled for the effect of BMI. Women with diabetes may be unaware of the difficulties achieving pregnancy, particularly if they have never experienced menstrual cycle disturbances. These findings have important implications regarding the potential medical treatment of women with diabetes who wish to have a child. Fertility drugs (commonly gonadotropins, gonadotropin-releasing hormone [GnRH] agonists and GnRH antagonists) are unlikely to affect the woman's glycaemic state [23]. Although current clinical management guidelines for women with pregestational diabetes discuss the role of preconception counselling for these women, the focus of these guidelines is on euglycaemic control as it relates to achieving the best possible pregnancy outcome [24]. In the future, it may be useful to have clinically relevant markers of hyperglycaemia among women with diabetes attempting pregnancy in order to aid in the elucidation of the mechanism by which diabetes affects fecundability. As with obese women or women with polycystic ovarian syndrome, protocols may be needed to better manage the care of women with diabetes who are planning a pregnancy.

Acknowledgements This research was supported in part by the Intramural Research Program of the National Institutes of Health, National Institute of Environmental Health Sciences. The Norwegian Mother and Child Cohort Study is supported by the Norwegian Ministry of Health, NIH/NIEHS (grant no N01-ES-85433), NIH/ NINDS (grant no.1 UO1 NS 047537-01), and the Norwegian Research Council/FUGE (grant no. 151918/S10).

Duality of interest The authors declare that there is no duality of interest associated with this manuscript.

\section{References}

1. Livshits A, Seidman DS (2009) Fertility issues in women with diabetes. Womens Health 5:701-707

2. Kjaer K, Hagen C, Sando SH, Eshoj O (1992) Epidemiology of menarche and menstrual disturbances in an unselected group of women with insulin-dependent diabetes mellitus compared to controls. J Clin Endocrinol Metab 75:524-529

3. Strotmeyer ES, Steenkiste AR, Foley TP Jr, Berga SL, Dorman JS (2003) Menstrual cycle differences between women with type 1 diabetes and women without diabetes. Diab Care 26:1016-1021

4. Solomon CG, Hu FB, Dunaif A et al (2001) Long or highly irregular menstrual cycles as a marker for risk of type 2 diabetes mellitus. J Am Med Assoc 286:2421-2426

5. Lorenzen T, Pociot F, Johannesen J et al (1999) A populationbased survey of frequencies of self-reported spontaneous and induced abortions in Danish women with type 1 diabetes mellitus. Diab Med 16:472-476

6. Melamed N, Hod M (2009) Perinatal mortality in pregestational diabetes. Int J Gynecol Obstet 104(Suppl 1):S20-S24

7. Ramosarroyo MA, Rodriguezpinilla E, Cordero JF (1992) Maternal diabetes - the risk for specific birth defects. Eur J Epidemiol 8:503-508

8. Jonasson JM, Brismar K, Sparen P et al (2007) Fertility in women with type 1 diabetes - a population-based cohort study in Sweden. Diab Care 30:2271-2276

9. Magnus P, Irgens LM, Haug K, Nystad W, Skjaerven R, Stoltenberg C (2006) Cohort profile: the Norwegian Mother and Child Cohort Study (MoBa). Int J Epidemiol 35:1146-1150 
10. Irgens LM (2000) The Medical Birth Registry of Norway. Epidemiological research and surveillance throughout 30 years. Acta Obstet Gynecol Scand 79:435-439

11. Weinberg CR, Baird DD, Rowland AS (1993) Pitfalls inherent in retrospective time-to-event studies: the example of time to pregnancy. Stat Med 12:867-879

12. Weinberg CR, Wilcox AJ (2008) Methodologic issues in reproductive epidemiology. In: Rothman KJ, Greenland S, Lash TL (eds) Modern epidemiology. Lippincott Williams \& Wilkins, Philadelphia, pp 620-640

13. Weinberg CR, Baird DD, Wilcox AJ (1994) Sources of bias in studies of time to pregnancy. Stat Med 13:671-681

14. Joffe M, Key J, Best N, Keiding N, Scheike T, Jensen TK (2005) Studying time to pregnancy by use of a retrospective design. Am J Epidemiol 162:115-124

15. Sutton-McDowall ML, Gilchrist RB, Thompson JG (2010) The pivotal role of glucose metabolism in determining oocyte developmental competence. Reproduction 139:685-695

16. Slocum JM (2007) Preconception counseling and type 2 diabetes. Diab Spectr 20:117-123

17. Pinto AB, Carayannopoulos MO, Hoehn A, Dowd L, Moley KH (2002) Glucose transporter 8 expression and translocation are critical for murine blastocyst survival. Biol Reprod 66:17291733

18. Yeshaya A, Orvieto R, Dicker D, Karp M, Ben-Rafael Z (1995) Menstrual characteristics of women suffering from insulin-dependent diabetes mellitus. Int J Fertil Menopausal Stud 40:269-273

19. Engeland A, Bjørge T, Daltveit AK, Vollset SE, Furu K (2009) Validation of disease registration in pregnant women in the Medical Birth Registry of Norway. Acta Obstet Gynecol Scand 88:1083-1089

20. Stene LC, Eidem I, Vangen S, Geir J, Lorentz MI, Narve M (2007) The validity of the diabetes mellitus diagnosis in the medical birth registry of Norway. Norsk Epidemiologi 17:165-174

21. Persson M, Norman M, Hanson U (2009) Obstetric and perinatal outcomes in type 1 diabetic pregnancies: a large, population-based study. Diab Care 32:2005-2009

22. Law DCG, Maclehose RF, Longnecker MP (2007) Obesity and time to pregnancy. Hum Reprod 22:414-420

23. No author listed (2008) Physicians' desk reference. Thomson PDR, Montvale

24. American College of Obstetricians and Gynecologists (2005) ACOG Practice Bulletin no. 60: pregestational diabetes mellitus. Obstetrics \& Gynecology 105:675-685 